\title{
Automated Visitor Record System
}

\author{
Fadhlan Hafizhelmi Kamaru Zaman ${ }^{\#}$, Ahmad Asari Sulaiman ${ }^{\#}$, Syahrul Afzal Che Abdullah", Mohd Fuad \\ Abdul Latip ${ }^{\#}$, Zairi Ismael Rizman ${ }^{\&}$ \\ ${ }^{\#}$ Department of Computer Engineering, Faculty of Electrical Engineering, Universiti Teknologi MARA, Shah Alam, Selangor, Malaysia \\ E-mail:fadhlan@salam.uitm.edu.my,asari100@gmail.com,bekabox@gmail.com,mamoncang@gmail.com \\ ${ }^{\&}$ Faculty of Electrical Engineering, Universiti Teknologi MARA, Dungun, Terengganu, Malaysia \\ E-mail: zairi576@tganu.uitm.edu.my
}

\begin{abstract}
As normally practiced, the information of visitor is recorded manually by either the visitor or the guard when the visitor arrives at the guard house. This manual process takes large amount of time and involves tedious works. Furthermore, it is prone to fraudulent information provided by visitors and occasionally interferes with guard's actual job in securing the area. This paper aims to provide an automated solution to these aforementioned problems. We address these problems by replacing the existing manual way of recording visitor information by an automated and fully computerized system. The solution proposed uses Radio-Frequency Identification (RFID), smart card information retrieval as well as computer vision and image processing to record and manage visitors' data. To evaluate the similarity between face images from camera and National Registration Identification Card (NRIC), we propose a method to find dissimilarity index between the faces. We found that the system minimizes the need for human interventions, improves the time required during recording of visitors' information as well as efficiently manages and analyses visitors' records. Additionally, our proposed method for finding face similarity yields a promising result of TPR $=0.914$ and FPR $=0.140$ when tested using publicly available face dataset called AR Dataset. The system is able to minimize the need for guard and visitor interventions, improves the time required during recording of visitors' information especially for recurring visits and also capable of securely and efficiently manages and analyses visitors' records.
\end{abstract}

Keywords — automated systems; computer vision; face similarity; RFID technology.

\section{INTRODUCTION}

Currently, the information of visitor is recorded manually when they arrive at the reception desk or at the guard house. The particulars acquired for records include identity card number, name, telephone number, address and the purpose of the visit. This manual process takes large amount of time and involves tedious works. Moreover, manual recording would be problematic when we need to reorganize, retrace and analyse the record especially in case of emergency. There is also an increasing concern over manual record system which interfere guards' attention on secured area. Thus, we believe that we can address aforementioned problem by replacing the existing manual way of recording visitor information by an automated, fully computerized system.

Some previous work on visitor record system includes Electronic visitor information system (E-VIMS) [1]. Limitation of E-VIMS is visitor without a valid NRIC cannot register into the system and there is no verification method employed in the system, thus can compromise the security of the premise. It also lacks any analysis on the visitor information or certain visit patterns. Similarly, another system employs face detection algorithm [4] but it also lacks verification and data analysis [2], [3]. Some other existing system are Visitor Management System (VMS) [4], museum ticketing and visitor management systems [5] which uses RFID technology which is capable of providing authorization [6] and visitor monitoring system which employs face recognition [7].

Currently, the most popular object matching method is Scale-Invariant Feature Transform (SIFT) features since they can match images under different scales, rotations and lighting. Some recent implementations of SIFT can be found in previous researches [8-10]. SIFT features rely on the fact that certain parts of an image have more information than others to match images, these are the features that is important for matching process. Similar implementation of matching key points is also employed [11]. Some recent method for image matching which uses paper cards and Convolutional Neural Network (CNN) that uses Regionbased Convolutional Network (R-CNN) [12], [13]. On the other hand, histograms can also be used for finding image similarity [14-16]. 
This paper proposes a biometrics-based visitor management system called Automated Visitor Record System (AVRS) which is able to record and carry out data analysis, efficiently monitors visitor's information and provides quick report generation. The data recording process begins when the visitor hands out his/her National Registration Identity Card (NRIC) or MyKad [17]. Using a smart card reader device, all the information in the identity card is automatically acquired and stored into a centralized server, taking note the current time. A photo of the visitor's face is taken simultaneously, which utilizing face detection algorithm for first-time record. Biometrics face verification then acts as added security measure and for faster process of record retrieval. A Radio-Frequency Identification (RFID) visitor card will be issued during check in and the identity card will be returned when the visitor checks out. The checkout time will also be updated accordingly.

As an added security measure, we propose an additional feature to be incorporated into this system, where a face similarity detection algorithm will try to gauge the similarity between face image from the camera and the one provided by the visitor in the NRIC. Face similarity detection is part of image matching algorithm, where this is actually a fundamental aspect of computer vision [18]. This feature is very important to provide ample protection against any fraudulent information from ill-intentioned visitor, especially in sensitive places such as police stations and government offices.

We found that this system minimizes the need for human interventions and also promotes green technology, since it involves paperless record keeping. It also improves the flow of visitor registration at receptionist desks or guard houses. Additionally, the system keeps the relevant data synchronized online and enables real time access at other entrances. Moreover, it aids the guard to actually focus on keeping the parameters secured rather than spending too much time on paperwork. Nevertheless, the face similarity detection also provides higher degree of security and confidence towards the ability of the proposed system.

\section{MATERIAL AND METHOD}

In this paper, we proposed two contributions that is the design of an automated visitor management system called AVRS which a method for detecting face similarity. These contributions are elaborated further in the following sections.

\section{A. Automated Visitor Record System}

Using Microsoft Visual Studio in C\# language, we have developed a .Net -based system which is capable of carrying multiple processes of registering and managing visitors in the premise. Essentially, the developed system is developed so that it can be capable of:

1. Checking visitors in and out with the use of simple RFID tagging and retrieval

2. Taking visitor's face image using active real-time face detection

3. Reading the visitor's information in the NRIC

4. Verify similarity between visitor's face and the one provided in the NRIC

5. Faster check-in for recurring visits with the use of automated data retrieval system

6. Manual check-in for visitors not having a valid NRIC

7. Data analysis with visit search, visit patterns statistics and visitor image search

8. User management system with privilege assignment and task-specific access

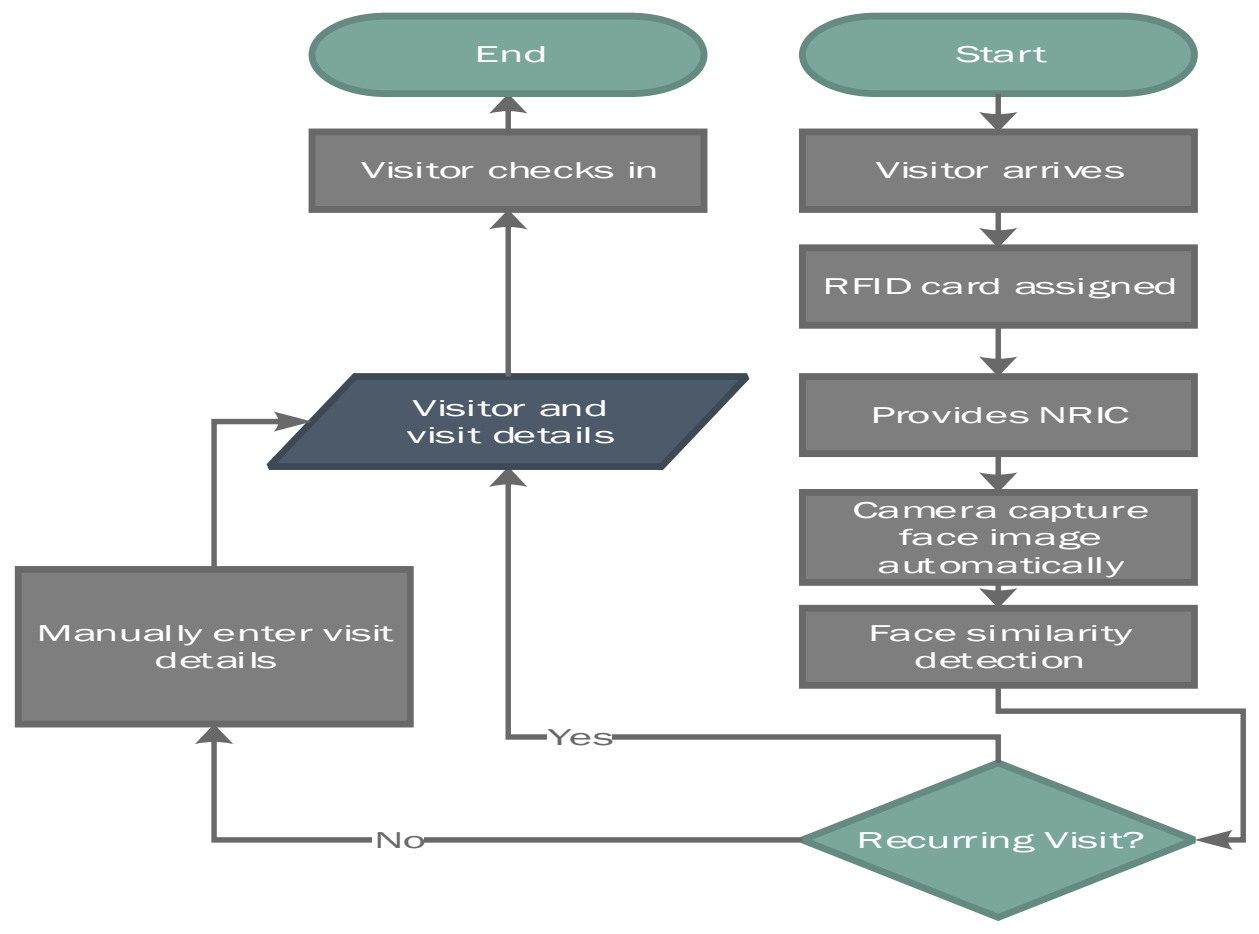

(a)

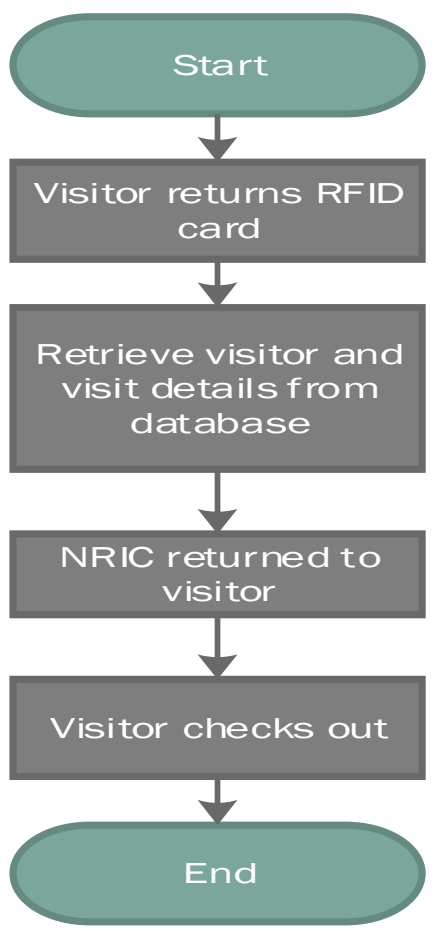

(b)

Fig. 1 The process of visitor (a) checks-in and (b) checks-out used in AVRS 
The process flow of visitor check-in and check-out is shown in Fig. 1. The check-in process begins when the visitor arrives at the guard house of reception desk. An RFID card is assigned to the visitor, while the visitor will provide his/her NRIC. The system will read the information from the NRIC including the NRIC number, address, face image and other relevant information while at the same time a camera will automatically detect the visitor face and later store his/her images in the database. The face detection algorithm used is based on Viola-Jones method [19]. Face similarity detection is then carried out to determine the NRIC provided is a valid NRIC. For recurring visits, the visit data will be retrieved automatically thus reducing significantly the time required for registration. Finally, the visitor checks in and appropriate status and indicators are assigned to the visitor information.
For check-out process, the visitor will hand-over the RFID card and from the unique ID in the card, the visitor information can be retrieved. The visitor's NRIC will be returned and the check-out status is assigned accordingly. This process manages to significantly reduce the time for check-out as the location where the NRIC is placed can be explicitly saved along with visit information, thus reducing the time for locating the NRIC if the number of visitors in the premise is high. The actual hardware setup of AVRS proposed in this paper is given in Fig. 2.

The system is developed using Microsoft SQL Server which we deemed sufficient and capable of supporting this system. For the limitation of this server, it is elaborated here: https://docs.microsoft.com/en-us/sql/sql-server/maximumcapacity-specifications-for-sql-server.
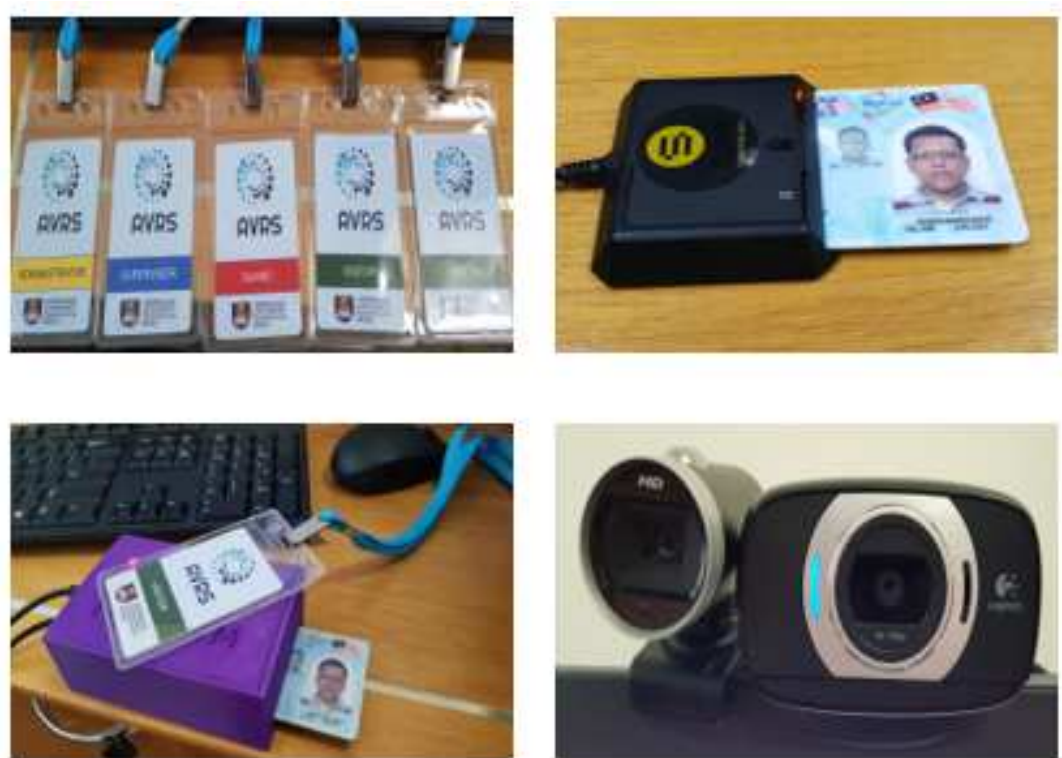

(a)

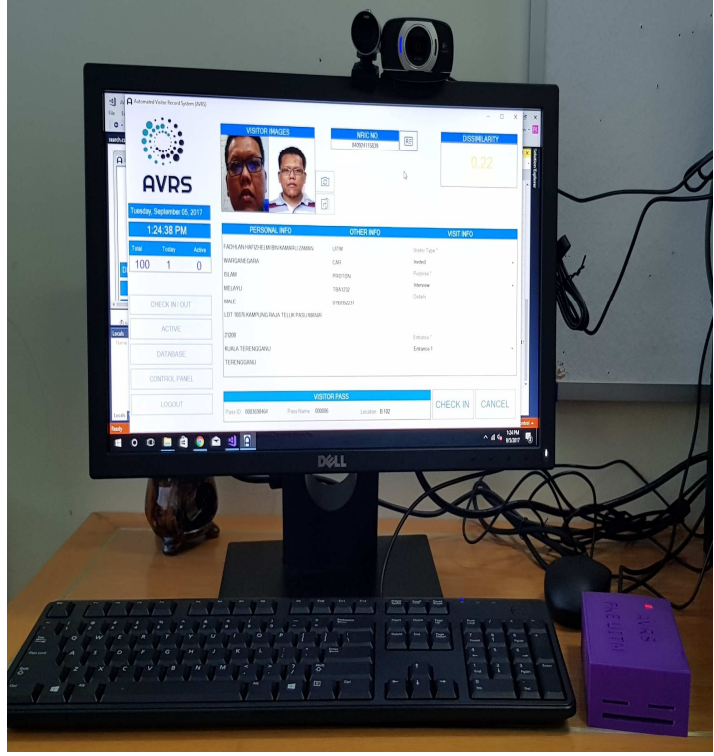

(b)

Fig. 2 The hardware used to build the system. (a) (following clock cycle) from top left is RFID cards, Smart card reader with an NRIC attached, 125KHz RFID reader and webcams. (b) The complete setup of the proposed system with its hardware and a PC

\section{B. Face Similarity Detection}

Face similarity detection can be defined as a process of detecting whether certain pair of face images called Face 1 (F) and Face $2(\mathrm{P})$ which belong to the same individual or not. Pair of images $\mathrm{F}$ and $\mathrm{P}$ can be classified into two classes (1) similar faces and (2) dissimilar faces, as depicted Fig. 3. Similar faces are two images which are not identical but it belongs to the same person, while dissimilar images are faces belong to different persons entirely.
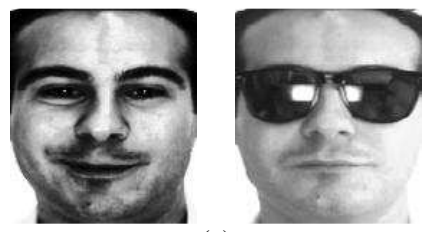

(a)

Fig. 3 Example of (a) similar and (b) dissimilar faces

In this paper, we propose a face similarity measure call face dissimilarity index (D) which indicates the dissimilarity between faces. Higher number of D would signify higher dissimilarity, thus higher chance for the face images to actually belong to different person. Prior to the computation of D, the local Gabor features of both faces are extracted following approach outlined [20]. The dissimilarity index (D) can be denoted as the total Cosine distance between $\mathrm{m}$ local patches (LP) of images, which given in Equation (1)

$\mathrm{D}=\sum_{\mathrm{m}=1}^{\mathrm{M}} \frac{\mathrm{P}_{\mathrm{m}} \cdot \mathrm{F}_{\mathrm{m}}}{\left(\left\|\mathrm{P}_{\mathrm{m}}\right\| \cdot\left\|\mathrm{F}_{\mathrm{m}}\right\|\right)}$

\section{RESULTS AND DISCUSSION}

The final AVRS system has been developed and several tests have been conducted specially to evaluate the performance of the proposed face similarity measure. In this section, the developed graphical user interface (GUI) of AVRS as well as the performance of the proposed face similarity measure is presented. 


\section{A. Automated Visitor Record System}

The GUI for visitor information is shown Fig. 4.

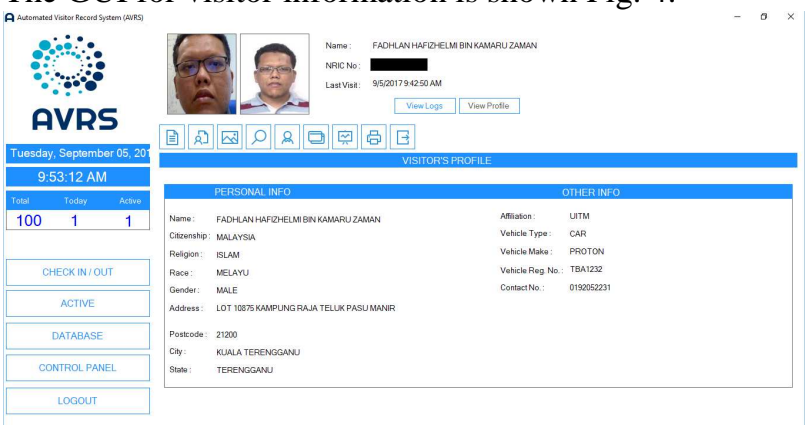

Fig. 4 GUI of AVRS showing the windows for displaying visitor's information

\section{B. Results on Face Similarity Detection}

The AR dataset contains frontal images of 76 males and 60 females with several types of variations [21]. Images were taken in two sessions with 13 images per session where 14 images per subject contain expressions and illumination variation, 12 images per subject depict the subject as wearing sunglasses and scarf. We use 100 subjects of 50 males and 50 females for the experiment following procedures [22]. All images used are aligned to $98 \times 98$ pixels. Then, histogram equalization is applied on all images. Subsequently, the intensities of all images are normalized using local normalization filter. Similarly, in order to acquire Gabor images from normalized images, the values of parameters that be used are $0 \leq \mathrm{v} \leq 4,0 \leq \mathrm{u} \leq 7, \mathrm{f}^{\mathrm{v}}=$ $\sqrt{2} .7, \mathrm{k}_{\max }=\pi / 2$ and $\sigma=\pi$ where $\mathrm{z}, \mathrm{u}, \mathrm{v}, \mathrm{f}, \mathrm{k}_{\max }$ is the pixel, orientation, scale, step in frequency and maximum frequency respectively. Using the locally lateral subspace to prepare the local Gabor features and our proposed method to find dissimilarity index, we evaluate the distance between images in AR database to obtain the distribution of face dissimilarity index [23]. The histogram of D for both similar faces and dissimilar faces is given in Fig. 6.

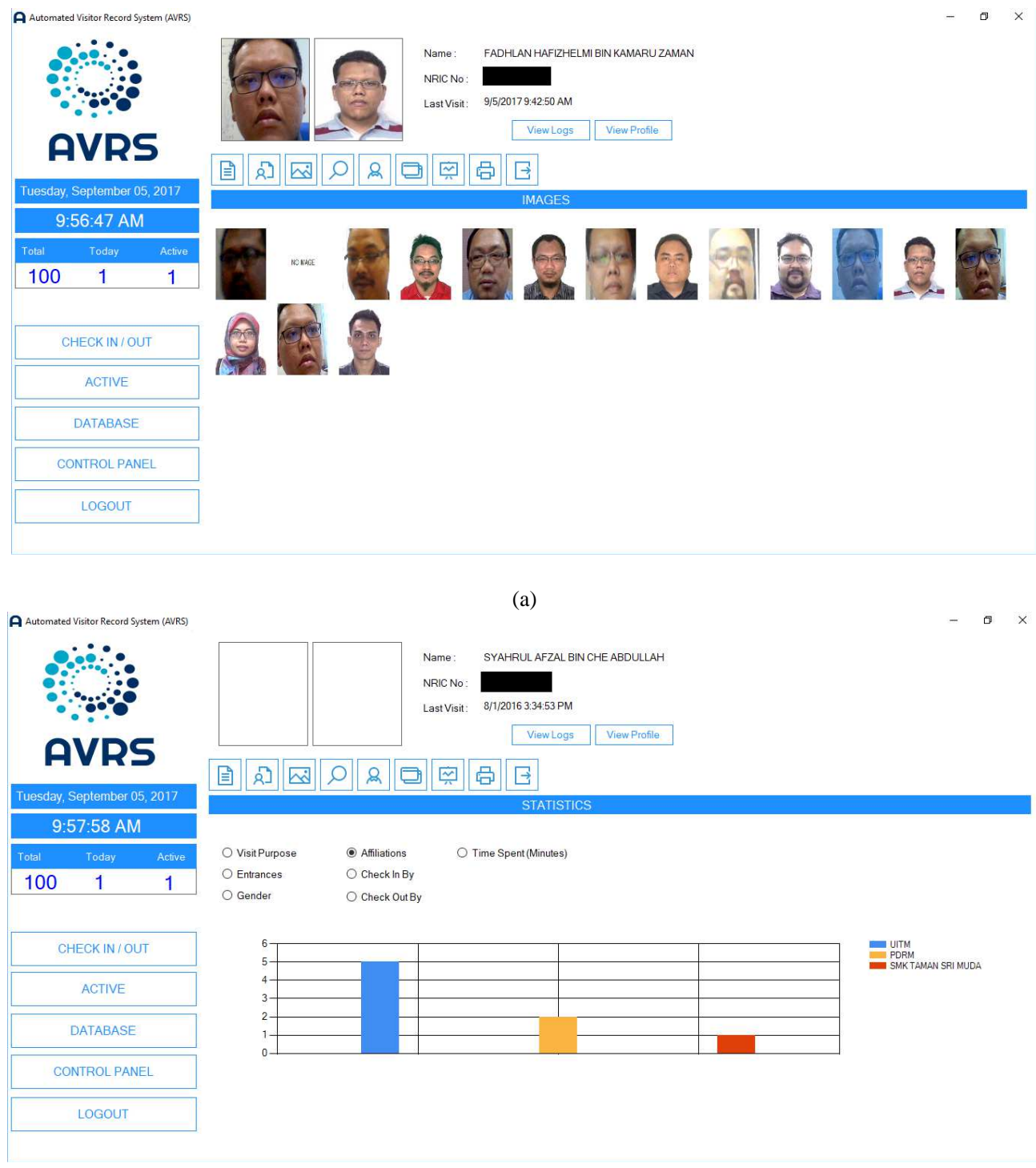

(b)

Fig. 5 GUI of AVRS showing the windows for (a) displaying visitor's images search (b) statistics windows for different types of affiliations and 
From Fig. 6, the distribution of D across the similar and dissimilar faces tend to overlap between $\mathrm{D}=5$ to $\mathrm{D}=9$ where faces having $\mathrm{D}$ around these values can either be similar or dissimilar. For example, let say we choose $D=5$ as the threshold, when similar pair of images having $D=6$, it will be incorrectly classified as dissimilar while a dissimilar pair of images having $\mathrm{D}=4$ will be incorrectly classified as similar. To obtain the best classification result, it is important to set the threshold so that it can maximize True Positive Rate (TPR) while minimizing False Positive Rate (FPR). Here the TPR denotes the rate at which similar faces are correctly classified as being similar to each other, while FPR denotes the rate at which dissimilar faces are incorrectly classified as being similar to each other.

Thus, from Receiver Operating Characteristics (ROC) curve shown in Fig. 7, the best threshold of dissimilarity index between similar and dissimilar faces is found to be D $=7$ (as denoted in Fig. 7) For instance, faces having D $\leq 7$ will be classified as similar, while faces having $D>7$ will be classified as dissimilar. Furthermore, the ROC for D $=7$ yields a promising result at TPR $=0.914$ and $\mathrm{FPR}=0.140$ respectively. The resulting TPR and FPR gives high probability of correctly classifying similar faces, while the low FPR will ensure low probability of incorrectly classifying dissimilar faces as being similar.

This result indicates that similar and dissimilar faces in fact shares some non-discriminatively low-level features that may contribute to misclassification of them as belong to similar faces. Carefully selecting those features and ranking them or even removing them might produce better results for this algorithm, such as using Linear Discriminant Analysis (LDA) [24]. However, for the purpose of AVRS development, it requires faster algorithm such as proposed, and thus we chose $\mathrm{D}=7$ for the development of the system and we omit any feature ranking or feature discrimination methods.

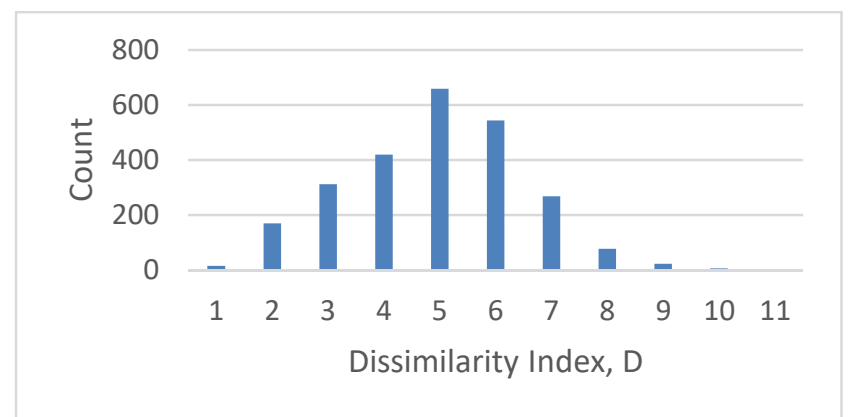

(a)

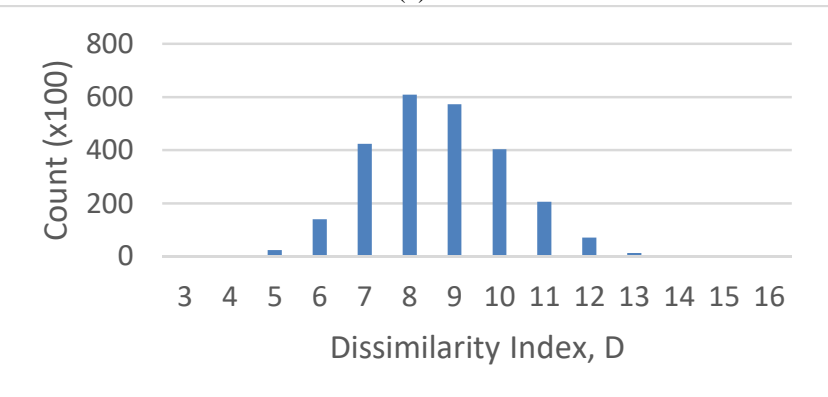

(b)

Fig. 6 The histogram distribution of dissimilarity index (shown as x-axis) for (a) similar faces and (b) dissimilar faces

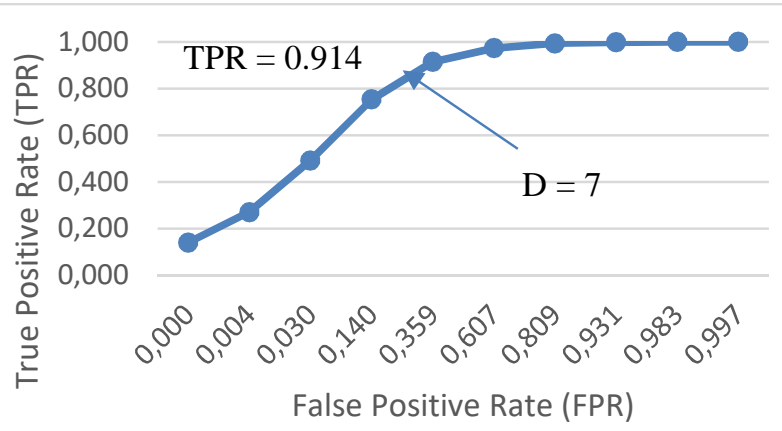

Fig. 7 The ROC of the proposed face similarity comparison based on the dissimilarity index classified using different thresholds

We then proceed to measure average time required to perform various activities using AVRS system. These activities are performed 20 times and the average time are recorded. This is shown in Table 1. From Table 1, we can see the improvement in terms of waiting time required while checking in visitors as compared to manual process. The time required for new check-in is 35 seconds while manual check-in using conventional method requires around 150 seconds due to manual typing of visitor's details. For recurring check-in, AVRS cut down the time further to just 8 seconds by recalling the stored data from the database.

TABLE I

AVERAGE TIME REQUIRED FOR VARIOUS ACTIVITIES

\begin{tabular}{|l|l|}
\hline Activity & Average Time Required (s) \\
\hline AVRS Check-in (New) & 35 \\
\hline AVRS Check-in recurring & 16 \\
\hline Check-in (without AVRS) & 150 \\
\hline AVRS Check-out & 8 \\
\hline
\end{tabular}

\section{CONCLUSION}

In this paper, we presented an automated system called AVRS specifically developed to improve the existing visitor's information management mechanism at guard houses or reception desks. We found that the AVRS is able to minimize the need for guard and visitor interventions, reduces the waiting time during recording of visitors' information especially for recurring visits and also capable of efficiently manages and analyses visitors' records. Moreover, as added security measure, we propose in this paper a method for finding similarity between faces using dissimilarity index which yields a promising result where we acquired the best TPR and FPR of 0.914 and 0.140 respectively for the ROC of the system. As future work, the system can be improved further by incorporating additional biometrics feature verification such as by using fingerprints besides the existing face verification for added security especially at sensitive places like police station and governments premises. In term of software performance, more rigorous test on application memory management and server stress test should be conducted to ensure the system is able to deliver optimal performance.

\section{ACKNOWLEDGMENT}

The authors would like to thank Dr. Aleix M. Martinez for providing the AR Face Dataset. The work presented here is 
sponsored by Universiti Teknologi MARA, Malaysia under grant 600-RMI/DANA 5/3/LESTARI (20/2015).

\section{REFERENCES}

[1] M. N. Noorhuzaimi, S. Junaida, A. Noraziah, and K. H. Chen, "Evisitor information management system (E-VIMS) using MyKad," in Proc. ICADIWT'08, 2008, p. 44

[2] I. M. Yassin, A. Zabidi, M. S. A. M. Ali, N. M. Tahir, H. A. Hassan, H. Z. Abidin, and Z. I. Rizman, "Binary particle swarm optimization structure selection of nonlinear autoregressive moving average with exogenous inputs (NARMAX) model of a flexible robot arm," International Journal on Advanced Science, Engineering and Information Technology, vol. 6, pp. 630-637, Oct. 2016.

[3] M. N. M. Nor, R. Jailani, N. M. Tahir, I. M. Yassin, Z. I. Rizman, and R. Hidayat, "EMG signals analysis of BF and RF muscles in autism spectrum disorder (ASD) during walking," International Journal on Advanced Science, Engineering and Information Technology, vol. 6, pp. 793-798, Oct. 2016.

[4] N. Anwar, M. N. Masrek, and Y. R. Rambli, "Visitor management system by applying the model of UTAUT," in Proc. IEEE ISBEIA'12, 2012, p. 223.

[5] S. Xiwen, "RFID technology-based museum ticketing and visitor management systems," in Proc. ICIMIMIE'13, 2013, p. 304.

[6] S. Kumari, "Real time authentication system for RFID applications," Indian Journal of Science and Technology, vol. 7, pp. 47-51, Mar. 2014.

[7] B. S. Satari, N. A. A. Rahman, and Z. M. Z. Abidin, "Face recognition for security efficiency in managing and monitoring visitors of an organization," in Proc. ISBAST'14, 2014, p. 95.

[8] W. L. Zhao and C. W. Ngo, "Flip-invariant SIFT for copy and object detection," IEEE Transactions on Image Processing, vol. 22, pp. 980-991, Mar. 2013.

[9] Y. Li, J. Zhou, A. Cheng, X. Liu, and Y. Y. Tang, "SIFT keypoint removal and injection via convex relaxation," IEEE Transactions on Information Forensics and Security, vol. 11, pp. 1722-1735, Aug. 2016.

[10] X. Pan, C. Nian, T. Bochao, W. Shaowei, and W. Han, "Efficient SIFT descriptor via color quantization," in Proc. ICCEC'14, 2014, p. 1 .

[11] A. Kelman, M. Sofka, and C. V. Stewart, "Keypoint descriptors for matching across multiple image modalities and non-linear intensity variations," in Proc. IEEE CCVPR'07, 2007, p. 1.
[12] S. Banerjee, J. Sweet, C. Sweet, and M. Lieberman, "Visual recognition of paper analytical device images for detection of falsified pharmaceuticals," in Proc. IEEE WACV'16, 2016, p. 1.

[13] R. Girshick, J. Donahue, T. Darrell, and J. Malik, "Region-based convolutional networks for accurate object detection and segmentation," IEEE Transactions on Pattern Analysis and Machine Intelligence, vol. 38, pp. 142-158, Jan. 2016.

[14] K. Lee, C. Lee, S. A. Kim, and Y. H. Kim, "Fast object detection based on color histograms and local binary patterns," in Proc. IEEE TENCON'12, 2012, p. 1.

[15] Z. Hongming, G. Wen, C. Xilin, and Z. Debin, "Learning informative features for spatial histogram-based object detection," in Proc. IEEE IJCNN'05, 2005, p. 1806.

[16] R. Palmer, G. West, and T. Tan, "Scale proportionate histograms of oriented gradients for object detection in co-registered visual and range data," in Proc. DICTA'12, 2012, pp. 1.

[17] R. C. W. Phan and L. A. Mohammed, "On the security and design of MyKad," in Proc. APCC'03, 2003, p. 721.

[18] D. G. Lowe, "Distinctive image features from scale-invariant keypoints," International Journal of Computer Vision, vol. 60, pp. 91-110, Nov. 2004.

[19] P. Viola and M. Jones, "Rapid object detection using a boosted cascade of simple features," in Proc. IEEE CVPR'01, 2001, p. 511.

[20] F. Kamaruzaman and A. A. Shafie, "Recognizing faces with normalized local Gabor features and spiking neuron patterns," Pattern Recognition, vol. 53, pp. 102-115, May 2016.

[21] A. M. Martinez and R. Benavente, "The AR face database," Computer Vision Center, Barcelona, Spain, CVC Technical Report, 1998.

[22] A. M. Martinez, "Matching expression variant faces," Vision Research, vol. 43, pp. 1047-1060, Apr. 2003.

[23] F. K. Zaman, A. A. Shafie, and Y. M. Mustafah, "Robust face recognition against expressions and partial occlusions," International Journal of Automation and Computing, vol. 13, pp. 319-337, Aug. 2016.

[24] G. Lange, C. Y. Low, K. Johar, F. A. Hanapiah, and F. Kamaruzaman, "Classification of electroencephalogram data from hand grasp and release movements for BCI controlled prosthesis," Procedia Technology, vol. 26, pp. 374-381, Dec. 2016. 